\begin{tabular}{|c|c|c|}
\hline 150 (150) & $\begin{array}{c}\text { Bulletin of Pharmaceutical Sciences } \\
\text { Assiut University }\end{array}$ & 6 \\
\hline 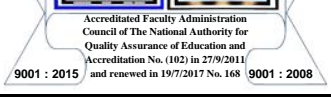 & $\begin{array}{l}\text { Website: http://bpsa.journals.ekb.eg/ } \\
\text { e-mail: bullpharm@aun.edu.eg }\end{array}$ & $\begin{array}{l}\text { BULLL. PHARM. SCI. } \\
\text { Assiut Univ. }\end{array}$ \\
\hline
\end{tabular}

\title{
ROLE OF MUCOSAL-ASSOCIATED INVARIANT T CELLS IN SYSTEMIC LUPUS ERYTHEMATOSUS
}

\author{
Rehab M. Mekkawy ${ }^{1 *}$, Omnia El-badawy ${ }^{2}$, Helal F. Hetta ${ }^{2,3}$, Doaa K. Abd El-hafez ${ }^{4}$ and \\ Khaled M. Hassanein ${ }^{2}$ \\ ${ }^{1}$ Forensic Medicine Authority, Ministry of Justice, Egypt. \\ ${ }^{2}$ Medical Microbiology and Immunology Department, Faculty of Medicine, Assiut University, \\ Assiut 71515, Egypt. \\ ${ }^{3}$ Department of internal Medicine, College of Medicine, University of Cincinati, Cincinati, OH \\ 45267-0595, USA. \\ ${ }^{4}$ Physical Medicine, Rheumatology and Rehabilitation Department, Faculty of Medicine, \\ Assiut University, Assiut, Egypt.
}

\begin{abstract}
The etiology of inflammatory and autoimmune diseases is complex and not yet fully understood. Their pathogenesis includes mechanisms in which both innate and adaptive immune cells are involved. Mucosal-associated invariant T (MAIT) cells are immune cells

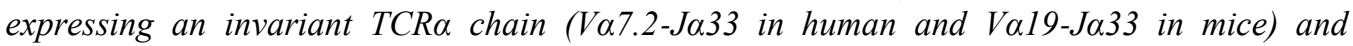
recognize the conserved MHC-I-related molecule MR1, which presents bacterial metabolites derived from the synthesis of vitamin B. Although most of the studies support a hazardous role of MAIT cells in tissue inflammation and destruction, few studies suggest a protective role. MAIT cells have tissue homing properties with the production of inflammatory cytokines, signifying that they may play an essential role in autoimmune and inflammatory diseases. In the current study, we clarified the present knowledge on MAIT cells in systemic lupus erythematosus.
\end{abstract}

\section{INTRODUCTION}

Mucosal-associated invariant $\mathrm{T}$ (MAIT) cells are defined as a subset of innate-like $\mathrm{T}$ lymphocytes first described in $1999^{1}$. They are present abundantly in humans and have the property of rapidly expressing a range of proinflammatory cytokines. MAIT cells differ from conventional $\mathrm{T}$ cells in that they express an $\alpha \beta$ T-cell receptor (TCR) that has a limited TCR diversity, mostly including a semiinvariant TCR- $\alpha$ chain associated with a limited TCR- $\beta$ chain repertoire ${ }^{2}$ Another difference is that MAIT cells are not MHCrestricted but are restricted by the non-classical antigen-presenting molecule; class $1 \mathrm{~b}$ MHCrelated protein $1(\mathrm{MR} 1)^{3 \& 4}$. MR 1 acts by presenting non-protein antigens which contain precursors and derivatives from highly conserved riboflavin and folic acid metabolism biosynthetic pathways in bacteria, mycobacteria, and yeast ${ }^{5 \& 6}$.

MAIT cells were initially named after their unique location in the gut lamina propria. A previous study reported that germ-free mice lack MAIT cells, indicating their link with mucosal immunity ${ }^{3}$. A monoclonal antibody recognizing human Va7.2 TCR was produced in 2009 by Martin et ll. $^{7}$ and showed that $\mathrm{V} \alpha 7.2 \mathrm{TCR}+$ cells with high expression of CD161 were MAIT cells. MAIT cells are abundant in peripheral human blood and constitute up to $10 \%$ of blood CD3+ cells. The level of iNKT cells in human peripheral blood represents $0.01-1 \%$, and MAIT cell frequency is 10-1,000 folds more than iNKT cells.

Although little is known about the roles that MAIT cells play in disease, there is a

Received in 17/4/2021 \& Accepted in 16/6/2021 
growing understanding of these cells' role in host protection from intracellular pathogens. Many features suggest potential importance to immune-mediated pathology. MAIT cells display an intrinsic effector memory phenotype i. e. without the need for previous clonal expansion ${ }^{8}$. They can rapidly secrete a range of pro-inflammatory cytokines, including interferon- $\gamma$ (IFN- $\gamma$ ), tumor necrosis factor- $\alpha$ (TNF-a), interleukin-17 (IL-17), and the type 2 cytokine IL-4 on TCR ligation ${ }^{9 \& 10}$.

Systemic lupus erythematosus (SLE) is an autoimmune disease with multi-system involvement, factors like genetic susceptibility, environmental triggers including infections, and dysregulation of innate and adaptive immunity can predispose to the disease ${ }^{11 \& 12}$.

Within tissues, the local cytokine production by MAIT cells could lower the quantitative threshold of immune signaling, which is believed to affect the genetic predispositions that contribute to the initial pathogenesis in SLE. Also, several non-MHC loci contribute to the genetic predisposition to $\mathrm{SLE}^{12}$, so polymorphisms in MR1 or other MAIT cell-related genes could lead to such predispositions. Till now, these have not been described. Cho et al. (2014) reported a decrease in the level of MAIT cells in peripheral blood of patients with SLE and rheumatoid arthritis ${ }^{13}$, mainly in the CD8+ and double-negative subsets, which correlated with disease activity scores in both diseases. Also, the frequencies of MAIT cells secreting IFN- $\gamma$ (though not IL17 or IL-4) were reduced in peripheral blood in SLE.

Because of the disease's unpredictable nature, SLE patients still suffer from disease flares, Conventional biomarkers are suboptimal in detecting flares, its sensitivity $50 \%$, and specificity is $75 \%{ }^{14 \& 15}$. Multiple studies showed that interferon (IFN) signaling pathway had an essential role in SLE. A high level of IFN in the serum of patients with SLE had been shown in previous studies ${ }^{16 \& 17}$. Recently, gene expression studies showed an overexpression of IFN-stimulated genes (ISGs) in the blood of SLE patients, which indicated the significant role of IFN in $\mathrm{SLE}^{18 \& 19}$. Another study on ISG products has recognized IFN regulated chemokines and established the correlation of these chemokines with disease activity making them promising biomarker candidates ${ }^{20}$. One of the IFN-regulated chemokines that has the strongest correlation with SLE disease activity is IFN gamma inducible protein 10 (IP-10) or chemokine ligand 10 (CXCL10) ${ }^{20}$.

Markers of disease activity, including antidouble-stranded DNA antibodies (dsDNA antibodies), erythrocyte sedimentation rate (ESR), and C3 levels, are widely used but several patients present obvious disease-related signs and symptoms with normal or almost average values for these parameters. For example, in more than $40 \%$ of SLE patients, anti-dsDNA antibodies were not detected ${ }^{21}$, Also up to $15 \%$ of clinically - asymptomatic patients had high anti-dsDNA antibody titers ${ }^{22}$, ${ }^{23}$. Furthermore, some patients with central nervous system lupus or high SLEDAI had low anti-dsDNA antibody titers. ${ }^{24}$. Therefore, there is a need for new markers to predict and monitor disease activity. Increased classical signs of T-cell activity, such as the level of soluble interleukin-2 receptor (IL-2R) in serum $^{25,26}$, or upregulation of HLA-DR and IL$2 \mathrm{R}$ on circulating $\mathrm{T}$ lymphocytes ${ }^{27 \& 28}$, have been reported that make them have a pivotal role in the immune response. Up till now, HLA-DR expression on T-cell subsets in SLE patients has never been studied in a large cohort and has never been compared with other conventional parameters ${ }^{29}$.

\section{MAIT Cells}

Although most $\mathrm{T}$ cell studies are focusing on 'conventional' $\mathrm{CD} 4+$ and $\mathrm{CD} 8+\mathrm{T}$ cells that distinguish peptides presented by MHC class II or I, there are several prominent populations of $\mathrm{T}$ cells referred to as "unconventional" $\mathrm{T}$ cells that recognize non-peptide antigens presented by specialized MHC class I-like molecules ${ }^{8 \& 30}$. These MHC class I-like molecules are monomorphic, and the $\mathrm{T}$ cells that recognize them have unique and conserved TCR repertoires, and consisting of an invariant TCR $\alpha$-chain paired with a restricted TCR $\beta$-chain repertoire that differs from the highly polymorphic MHC class I molecules that present antigen to a diverse population of $\mathrm{T}$ cells $^{2}$. Also, these cells have unique effector functions by the recognition of these MHC class I-like targets during intrathymic development confers on the cells' developmental pathways that diverge from those of their peptide-MHC-restricted 
counterparts. So, their distinct TCRs and antigenic targets are central to the biology of these cells. Invariant TCR $\alpha$-chains in humans were first described in 1993 when a study of human CD4-CD8- T cells discovered two distinct TCR $\alpha$ sequences ${ }^{2}$. One was the $\alpha$-chain variable region 24 and $\alpha$-chain joining region 18 (V $224 \mathrm{~J} \alpha 18)$ that defines type I natural killer $\mathrm{T}$ cells (NKT cells) restricted to the antigenpresenting molecule $\mathrm{CD} 1 \mathrm{~d}^{8 \& 30}$, while the other included Va7.2 (TRAV1-2) joined to Ja33 (TRAJ33). Lantz and colleagues identified a population of $\mathrm{T}$ cells in mice and humans carrying the same TCR $\alpha$-chain ${ }^{1}$. In mice, these $T$ cells expressed the orthologous Va19Ja33 (TRAV1 TRAJ33), paired with a limited array of TCR $\beta$-chains, mainly V $\beta 6$ (TRBV19) and V $\beta 8$ (TRBV13), or V $\beta 2$ (TRBV20) and V $\beta 13$ (TRBV6) in humans ${ }^{1}$. The nomenclature of "mucosal-associated invariant $\mathrm{T}$ cells "(MAIT) cells is because they are enriching mucosal locations such as gut ${ }^{3}$. Moreover, MAIT cells are restricted to the non-polymorphic MHC class I-like protein MR1 ${ }^{3}$, a $\beta 2$-microglobulinassociated antigen-presenting molecule ${ }^{31}$. This interaction is largely deduced by the fact that MR1 and TRAV1 are highly preserved during the course of mammalian progress and have coevolved, with species that lack MR1 also selectively lacking TRAV $1^{32-34}$. MAIT cells are very abundant in non-mucosal human tissues in which they represent up to $10 \%$ of blood $\mathrm{T}$ cells $^{35 \& 36}$ and $45 \%$ of liver $T$ cells ${ }^{37}$.

\section{Antigens detected by MAIT cells}

Studies have shown that different MR1 ligands may induce several effects on MAIT cells. Riboflavin (vitamin B2)-derivatives were observed to activate MAIT cells ${ }^{38}$, but folate (vitamin B9)-derived antigens do not activate them. Riboflavin derivatives are pyrimidine products bound by MR1 of its unstable precursor, 5-Amino-6-D-Ribitylaminouraci (5A-RU). These compounds are produced by most of the prokaryotes and fungi but not in animals lacking the riboflavin biosynthesis pathway $^{39}$. Furthermore, some pathogenic bacteria cannot synthesize riboflavin and have adapted to its exogenous uptake. These explain the dependence of MAIT cell development on timely commensal colonization and the changes in MAIT cell activity observed in dysbiosis ${ }^{40}$. A small proportion of peripheral blood MAIT cells was revealed to be reactive towards the MR1 molecule itself ${ }^{41}$. Introducing MR1-Ag tetramers [loaded with 5-(2oxoethylideneamino)-6-D-ribitylaminouracil (5-OP-RU)] permit the determination of different phenotypical and functional MAIT cell subsets ${ }^{42} \& 43$. These antigens help in studing MAIT cells and in determination of the role of these cells in different diseases.

\section{Tools for studying MAIT cells}

Studying MAIT cells was done by recognizing antibodies specific for combinations of cell-surface markers, such as CD3, TRAV1-2 and CD161. Cells expressing these marker combinations are highly enriched in healthy human blood for MAIT cells, while it is uncertain how well these markers define these cells in other tissues or in the disease. The development of MR1 tetramers loaded with 5-OP-RU ${ }^{44,}{ }^{45}$ has a key advance in the study of MAIT cells, allowing new investigations and direct evaluation of the efficacy of surrogate phenotypes for the analysis of MAIT cells in humans ${ }^{36, \& 45 \& 46}$

Regarding MR1 tetramer staining, populations of blood-derived CD8+TRAV1-2+ T cells that express CD161, the dipeptidyl peptidase CD26 or the cytokine receptor CD218 in humans show considerable enrichment for MAIT cells, but not all of these are MAIT cells and not all MAIT cells are captured by this surrogate phenotype (11). Moreover, surrogate markers such as CD161 are not present on immature MAIT cells ${ }^{47}$ and may be downregulated on MAIT cells after activation ${ }^{48-50}$, which indicate how these cells act in disease states. MR1 tetramers have also instigated rapid advances in the study of mouse MAIT cells. First study of the phenotype and function of MAIT cells was investigated in 'normal' laboratory mice (i.e., without transgenic TCR expression) ${ }^{51}$, which was previously difficult because the antibodies specific for the Va19 (TRAV1) TCR $\alpha$-chain was not detected and MAIT cells are 10- to 100 -fold less frequent in mice than in humans ${ }^{1 \& 51 \& 52}$.

Other studies used mice with transgenic expression of an invariant TRAV1+TRAJ33+ TCR and MR1-knockout mice to study mouse MAIT cells ${ }^{7}, 53,54$. The development and function of TCR-transgenic cells may differ from that of non-transgenic MAIT cells as 
those TCR transgenic mice have large numbers of MAIT cells and have given valuable insight into the development and function of these cells. As, the TCR-transgenic MAIT cells ${ }^{7}$ mostly have a naïve phenotype and do not express the transcription factor PLZF (promyelocytic leukemia zinc finger), contrary to MAIT cells in non-transgenic mice ${ }^{51,52}$. Furthermore, TRAV1 + TRAJ33 + TCRtransgenic $\times \mathrm{TCR} \alpha$-chain constant region $(\mathrm{C} \alpha)$ knockout mice on an MR1-knockout background still develop a large population of MAIT like cells ${ }^{45 \& 55}$. A study revealed that the C57BL/6. CAST- congenic mouse strain produces about ten times more MAIT cells than does the non-congenic C57BL/6 mouse strain ${ }^{52}$. The MAIT cells in C57BL/6.CAST- congenic mice are PLZF + and MR1 dependent ${ }^{52}$. One more study has described TRAJ33- knockout mice $^{47}$. The determination of MR1 tetramer allow more specific study of MAIT cells in different fields.

\section{MAIT cell development}

MAIT cells developed in a thymusdependent manner ${ }^{1 \&} 7$ after random rearrangements in TCR-encoding genes. Cells that generate a TCR that interacts with MR1 expressed on CD4+CD8+ cortical thymocytes are selected into the MAIT cell lineage ${ }^{1 \& 7 \& 56}$. Like the CD1d-restricted type I NKT cells that also develop after interacting with CD4+CD8+ thymocytes and contrasts with conventional $\mathrm{T}$ cell development, during which positive selection requires interactions of the TCR with MHC molecules on thymic epithelial cells, which suggest that MAIT cells and type I NKT cells share many transcription factors and functions $^{57}$, as highlighted by transcriptomics comparisons of these cell types ${ }^{58 \& 59}$. But, there are some differences between MAIT cells and type I NKT cells; for example, most mouse MAIT cells produce the cytokine IL-17A, and only a small subset of these cells produce the cytokine IFN- $\gamma$, while the opposite is for type I NKT cells. Moreover, in mice, type I NKT cells are more than MAIT cells by 10- to 100fold in most tissues, while the opposite applies in humans. One common characteristic is that in contrast to conventional $\mathrm{T}$ cells, the number of MAIT cells and type I NKT cells varies widely between individual humans. Yet, the number of MAIT cells correlates with the number of type I NKT cells ${ }^{36}$ for reasons that remain unclear and with uncertain consequences for human immunity. The identification of immature MAIT cells and the mapping of MAIT cell development in both mice and humans was made by the use of MR1 tetramers to assess MAIT cells in the thymus ${ }^{47}$. Studing both MAIT cells and NKT cells help in the determination of their role in SLE disease.

\section{MAIT cell subsets}

MAIT cells were first discovered as a double negative (DN) CD4-CD8- $\mathrm{T}$ cell population rich in the peripheral blood of healthy people and identified by expressing an invariant $\mathrm{TCR} \alpha$ chain and the $\mathrm{CD} 161$ surface marker $^{2}$. These markers were used for identification and further phenotyping of human MAIT cells. MAIT cells express several surface molecules such as CD25, CD26, CD44, and CD69 surface markers also expresss cytokine receptors such as interleukin 7 receptor (IL-7R), IL-12R, IL-15R, and IL-18R also express transcription factors PLZF (promyelocytic leukaemia zinc finger), T-bet (T-box transcription factor) and ROR $\gamma \mathrm{t}$ (RARrelated orphan receptor gamma) ${ }^{60}$.

The majority of human peripheral blood MAIT cells were CD8+CD4- and about $14 \%$ of the peripheral blood MAIT cells were doublenegative. A minor subset was described as CD4+CD8- or CD4 + CD8 + double positive $^{41}$. These subsets differentially drop with age, signifying further alterations in their development regulation ${ }^{41}$. Different patterns of cytokine production were detected between the subsets $^{46}$. MAIT17 development was lately found to be strongly reliant on TCR signals in the thymus, being massively reduced in germfree mice. During colonization, maturation and expansion of thymic MAIT17 cells was detected, but MAIT1 cell numbers was not affected $^{61}$. Previous study confirms that the division into MAIT1 and MAIT17 subtypes occurs within the thymus and clarified other factors contributing to MAIT cell maturation and differentiation ${ }^{62,63}$. In human MAIT cells, no clear partition into MAIT1 and MAIT17 subtypes is observed, which are also strong producers of tumor necrosis factor $\alpha(\mathrm{TNF} \alpha)^{64}$. 


\section{MAIT cells in autoimmunity and inflammation}

Previous studies showed that in the peripheral blood of patients diagnosed with different disorders \{systemic lupus erythematosus ${ }^{13}$, ankylosing spondylitis ${ }^{65}$, rheumatoid arthritis (RA) $)^{49}$, vasculitis ${ }^{66}$, systemic sclerosis ${ }^{67}$, primary Sjögren syndrome $(\mathrm{pSS})^{68}$, primary sclerosing cholangitis (PSC) ${ }^{69}$ and inflammatory bowel disease ${ }^{70 \& 71}$, MAIT cells were significantly reduced. In Lupus, previous studies showed a strong positive correlation between MAIT cell deficiency and disease activity. Although, not all MAIT cell subsets decreased to the same extent. .For example, a change in the expression of CD4 and CD8 coreceptors was detected in RA and pSS patients $^{68}$.

MAIT cells infiltrate the tissues affected in the immune-mediated diseases. For example, Infiltration and downregulation of activation markers in the synovium of RA and spondyloarthritis patients, which is reliable with hyporesponsiveness to potent microbial stimuli $^{49}$. Furthermore, MAIT cells infiltrating biliary ducts in primary sclerosing cholangitis, salivary glands of pSS patients ${ }^{67}$, and intestinal lesions in inflammatory bowel disease ${ }^{70 \& 71}$. The migration of MAIT cells to the inflamed tissues may clarify the cause of why these cells decrease in the priephral blood of patients of different disorders in different studies.

\section{MAIT cells in cancer}

While evidence of the role of MAIT cells in cancer is limited, some studies point to a possible role for MAIT cells in tumor immunity. In an investigation of diverse tumor samples, KLRB1 (encoding CD161) was the gene most significantly associated with a good prognosis. This correlated with tumorinfiltrating $\mathrm{CD} 8+\mathrm{T}$ cells ${ }^{72}$, which may indicate that tumor-infiltrating MAIT cells may be a favorable prognostic factor.

\section{Systemic Lupus Erythematosus (SLE)}

Systemic lupus erythematosus is an idiopathic connective tissue disease. Although the etiology of SLE is presumed to be multifactorial, one of the disease characteristics is the production of autoantibodies which leads to immune complex deposition, inflammation, and permanent organ damage. SLE is one of the most known autoimmune disease of women of childbearing age (14.6 to 50.8 per 100,000 persons in this category in the USA) $)^{73-75}$. There is a female: male ratio of approximately 6-10:1, with a peak incidence between the ages of 15 and 40. On the other hand, SLE can affect all age groups, from infants to geriatric patients. Accurate clinical assessment of SLE is needed because it has a variable disease course, a complex phenotype, and cumulative morbidity over time. A new organ system may be involved over time in numerous patients, even 5-10 years after diagnosis ${ }^{76}$. Several studies now show 5-year survival rates exceeding $90 \%{ }^{77-79}$. Still, SLE patients' survival has not improved since the 1980s, with atherosclerosis remaining the major cause of death. Therefore, it is crucial to reveal new measures for diagnosing SLE, monitoring disease activity, assessing tissue damage, and recognizing effects on individual patients. ${ }^{80}$

\section{$T$ cells in SLE}

Particularly CD4 T-cells are of central importance, which can be found in the urine of patients with active lupus nephritis ${ }^{81}$. CD4+ T cells can occur in various subsets, such as follicular $\mathrm{T}$ helper cells ( $\mathrm{Tfh}$ ), regulatory $\mathrm{T}$ cells (Treg), Th17-, Th1- and Th2 cells. Tfh cells were elevated in SLE patients' peripheral blood and are essential for germinal centre induction, proliferation, isotype-switching and somatic hypermutation. These cells express both PD-1 and CXCR5 and produce the main cytokine IL-21, which provokes B cells to differentiate into memory $B$ cells and plasmablasts as typically found in $\mathrm{SLE}^{82,83}$. In lupus, increased numbers of Th-17 with an imbalance to reduced Tregs have also been reported $^{84}$. IL-17 mediates inflammation, and its production is inversely correlated with IL-2. By the way, blockade of IL-2 stimulates differentiation of Th17 cells ${ }^{85}$. IL-2 deficiency was described in SLE and represented an additional hallmark of this disease. Though, recent studies show that exogenous IL-2 cannot completely restore T-cell functions in $\mathrm{SLE}^{86}$.

\section{MAIT cells in SLE}

The MAIT cells play important roles in the first-line defense against microbial pathogenesis as they can respond very rapidly on activation, in the absence of exogenous 
antigens, by TCR signaling or cytokine stimulation, similar to other innate lymphocytes, including invariant natural killer $\mathrm{T}$ (iNKT) cells ${ }^{87-90}$. Several cytokines are produced by activated MAIT cells, including interferon (IFN)-g, TNF-a, and IL- 17 in addition to cytotoxic granzyme and perforin ${ }^{9 \& 35 \& 91 \& 92}$. MAIT cells activation is through the MR1 ligand present in microbes and drugs. Cytokines, can also activate MAIT cells including IL-12, IL-18, and IFN $\alpha$, by an antigen-independent mechanism. MAIT cells express several cytokine and chemokine receptors and homing receptors. Activated MAIT cells upregulate very late antigen-4 (VLA-4) and secrete perforin and granzyme-B, Th1 and Th17 cytokines, and and low levels of IL-10 (figure1) ${ }^{93}$.

MAIT cells are gaining attention because of their roles in various types of immune responses and diseases due to their characteristic abundance and cytokineproducing capacity. MAIT cells are related to an increasing number of diseases of microbial, autoimmune, metabolic, and cancerous origin ${ }^{94-}$ 96. A Previous study established that MR1 deficiency aggravated the severity of experimental autoimmune encephalomyelitis in an animal model of multiple sclerosis ${ }^{97}$, suggesting their protective role in this model because MR1 is necessary for the thymic development of MAIT cells (1-3). Further studies revealed that MAIT cells play role in the suppression of the severity of inflammatory colitis and the development of type 1 diabetes $^{98 \& 99}$. In contrast, MAIT cells seem to play role in tissue inflammation in arthritis models and in the pancreas of NOD mice after the onset of diabetes ${ }^{9 \& 99}$,

Systemic lupus erythematosus (SLE) is a multi-systemic autoimmune disease caused by the production of autoantibodies to nuclear antigens. Deposition of this autoantibodies in the form of immune complexes in tissues cause tissue inflammation in various organs, including the skin, joints, kidneys, and central nervous system. Although the new advances in the treatment of SLE, therapeutic options are still limited. A Preceding study revealed that the frequency of MAIT cells was reduced in the peripheral blood of patients with SLE, and the MAIT cells present were activated in SLE patients more than in healthy controls, and positively correlated with disease activity ${ }^{3}$. These findings suggest that MAIT cells may have an important role in lupus pathology. By crossing FcgRIIb-/-Yaa mice ${ }^{41}$, a spontaneous lupus mouse model, to MR1-deficient mice that lack MAIT cells, a previous study established that MAIT cell deficiency results in reduced disease severity by decreased autoantibody production, reduced glomerulonephritis scores and reduced germinal center responses in addition to lower $\mathrm{T}$ cell and innate $\mathrm{T}$ cell immune responses in MR1-deficient lupus mice.

A previous study revealed that the treatment of mice with new synthesized nonstimulatory MR1 ligand inhibits MAIT cell activation and reduced autoantibody production and the severity of lupus nephritis. Furthermore, it showed that MAIT cells enhanced autoantibody production by B cells in vitro dependent on CD40L-CD40 and TCR pathways. So, Inhibition of MAIT cell activation by using an inhibitory MR1 ligand reduced autoantibody production by $B$ cells ${ }^{100}$.In our study we try to explain the key role of MAIT cells in SLE disease make there is a possiblity of them as a therapeutic target in systemic autoimmune diseases, including SLE.

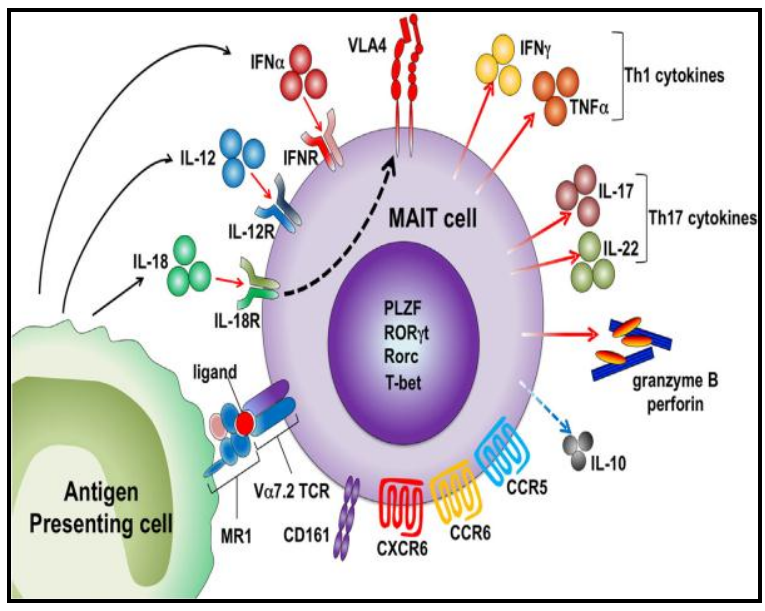

Fig. 1: Mechanisms of human mucosalassociated invariant $\mathrm{T}$ (MAIT) cells activation. ${ }^{93}$

\section{REFERENCES}

1. F. Tilloy, E. Treiner, S.H. Park, C. Garcia, F. Lemonnier, H. de la Salle, et al., "An invariant $\mathrm{T}$ cell receptor alpha chain 
defines a novel TAP-independent major histocompatibility complex class Ibrestricted alpha/beta $\mathrm{T}$ cell subpopulation in mammals", The Journal of experimental medicine, 189 (12), 19071921 (1999).

2. S. Porcelli, C. E. Yockey, M. B. Brenner and S.P. Balk, "Analysis of T cell antigen receptor (TCR) expression by human peripheral blood CD4-8- alpha/beta T cells demonstrates preferential use of several $\mathrm{V}$ beta genes and an invariant TCR alpha chain", The Journal of Experimental Medicine, 178 (1), 1-16 (1993).

3. E. Treiner, L. Duban, S. Bahram, M. Radosavljevic, V. Wanner, F. Tilloy, et al., "Selection of evolutionarily conserved mucosal-associated invariant $\mathrm{T}$ cells by MR1", Nature, 422(6928), 164-169 (2003).

4. Hinks TS, MR1 (in Mouse and Man), in: Ratcliffe M (Ed.), Encyclopedia of Immunobiology., Elsevier, Oxford, p. 3750, 2016.

5. L. Kjer-Nielsen, O. Patel, A.J. Corbett, J. Le Nours, B. Meehan, L. Liu, et al., "MR1 presents microbial vitamin B metabolites to MAIT cells", Nature, 491(7426), 717723 (2012).

6. S. B. Eckle, A. J. Corbett, A.N. Keller, Z. Chen, D.I. Godfrey, L. Liu, et al., "Recognition of Vitamin B Precursors and Byproducts by Mucosal Associated Invariant $\mathrm{T}$ Cells", The Journal of biological chemistry, 290(51), 3020430211 (2015).

7. E. Martin, E. Treiner, L. Duban, L. Guerri, H. Laude, C. Toly, et al., "Stepwise development of MAIT cells in mouse and human", PLoS biology, 7(3), 525-536, (2009).

8. D. I. Godfrey, A. P. Uldrich, J. McCluskey, J. Rossjohn and D. B. Moody, "The burgeoning family of unconventional T cells", Nature immunology, 16 (11), 1114-1123 (2015).

9. M. Dusseaux, E. Martin, N. Serriari, I. Péguillet, V. Premel, D. Louis, et al., "Human MAIT cells are xenobiotic- resistant, tissue-targeted, CD161hi IL-17secreting T cells", Blood, 117 (4), 12501259 (2011).

10. A. Rahimpour, A. Enders, R. Clanchy, S. B. Eckle, B. Meehan et al., "Identification of phenotypically and functionally heterogeneous mouse mucosal-associated invariant Tcells using MR1 tetramers",

The Journal of Experimental Medicine, 212, 1095-1108 (2015).

11. L. Lisnevskaia, G. Murphy and D. Isenberg, "Systemic lupus erythematosus", Lancet (London, England) 384(9957) 1878-1888 (2014).

12. M. Wahren-Herlenius and T. Dörner, "Immunopathogenic mechanisms of systemic autoimmune disease", Lancet (London, England), 382(9894), 819831(2013).

13. Y. N. Cho, S. J. Kee, T. J. Kim, H. M. Jin, M. J. Kim, H. J. Jung, et al., "Mucosalassociated invariant $\mathrm{T}$ cell deficiency in systemic lupus erythematosus", Journal of immunology, 193(8), 3891-3901 (2014).

14. A. Ho, S. G. Barr, L. S. Magder and M. Petri, "A decrease in complement is associated with increased renal and hematologic activity in patients with systemic lupus erythematosus", Arthritis and rheumatism, 44 (10), 2350-2357 (2001).

15. H. Julkunen, S. Ekblom-Kullberg and A. Miettinen, "Nonrenal and renal activity of systemic lupus erythematosus: a comparison of two anti-C1q and five antidsDNA assays and complement C3 and C4", Rheumatology international, 32 (8), 2445-2451 (2012).

16. S. R. Ytterberg and T. J. Schnitzer, "Serum interferon levels in patients with systemic lupus erythematosus", Arthritis and rheumatism, 25 (4), 401-406 (1982).

17. J. J. Hooks, H. M. Moutsopoulos, S. A. Geis, N. I. Stahl, J. L. Decker and A. L. Notkins, "Immune interferon in the circulation of patients with autoimmune 
disease", The New England journal of medicine, 301 (1), 5-8 (1979).

18. L. Bennett, A. K. Palucka, E. Arce, V. Cantrell, J. Borvak, J. Banchereau, et al., "Interferon and granulopoiesis signatures in systemic lupus erythematosus blood", The Journal of experimental medicine 197 (6), 711-723 (2003).

19. E. C. Baechler, F. M. Batliwalla, G. Karypis, P. M. Gaffney, W. A. Ortmann, K. J. Espe, et al., "Interferon-inducible gene expression signature in peripheral blood cells of patients with severe lupus", Proceedings of the National Academy of Sciences of the United States of America, 100 (5), 2610-2615 (2003).

20. J. W. Bauer, E. C. Baechler, M. Petri, F. M. Batliwalla, D. Crawford, W. A. Ortmann, $\boldsymbol{e t}$ al., "Elevated serum levels of interferon-regulated chemokines are biomarkers for active human systemic lupus erythematosus", PLoS medicine, 3 (12), 2274-2284 (2006).

21. J. G. Worrall, M. L. Snaith, J. R. Batchelor and D. A. Isenberg, "SLE: a rheumatological view. Analysis of the clinical features, serology and immunogenetics of 100 SLE patients during long-term follow-up", The Quarterly journal of medicine, 74 (275), 319-330 (1990).

22. P. H. Schur, J. Sandson, "Immunologic factors and clinical activity in systemic lupus erythematosus", The New England journal of medicine, 278 (10), 533-538 (1968).

23. U.M. Gladman DD and Keystone EC. "Serologically active clinically quiescent systemic lupus erythematosus: a discordance between clinical and serologic features", Am J Med, 66 (2), 210-215 (1979).

24. H. G. Bluestein, "Neuropsychiatric manifestations of systemic lupus erythematosus", The New England journal of medicine, 317, 309-311 (1987).
25. E. J. ter Borg, G. Horst, P. C. Limburg and C. G. Kallenberg, "Changes in plasma levels of interleukin-2 receptor in relation to disease exacerbations and levels of antidsDNA and complement in systemic lupus erythematosus", Clinical and experimental immunology, 82 (1), 21-26 (1990).

26. M. G. Danieli, P. Paoletti, A. Recchioni, A. Gabrielli and G. Danieli, "Serum levels of soluble interleukin-2 receptor in patients with systemic lupus erythematosus and systemic idiopathic vasculitis", Scandinavian journal of rheumatology, 22 (5), 215-219 (1993).

27. N. M. Raziuddin S, Al-Wabel AA, "Increased circulating HLA-DR1 CD41 T cells in systemic lupus erythematosus: alterations associated with prednisolone therapy", Scand J Immunol, 31 (2),139145 (1990).

28. P. E. Spronk, G. Horst, B. T. Van Der Gun, P. C. Limburg and C. G. Kallenberg, "Anti-dsDNA production coincides with concurrent $\mathrm{B}$ and $\mathrm{T}$ cell activation during development of active disease in systemic lupus erythematosus (SLE)", Clinical and experimental immunology, 104 (3), 446453 (1996).

29. J. F. Viallard, C. Bloch-Michel, M. NeauCransac, J. L. Taupin, S. Garrigue, V. Miossec, et al., "HLA-DR expression on lymphocyte subsets as a marker of disease activity in patients with systemic lupus erythematosus", Clinical and experimental immunology, 125 (3), 485491 (2001).

30. M. Salio, J. D. Silk, E. Y. Jones and V. Cerundolo, "Biology of CD1- and MR1restricted T cells", Annual review of immunology, 32, 323-366 (2014).

31. K. Hashimoto, M. Hirai, Y. Kurosawa, "A gene outside the human MHC related to classical HLA class I genes", Science (New York, N.Y.), 269(5224), 693-695 (1995). 
32. P. Riegert, V. Wanner, S. Bahram, "Genomics, isoforms, expression, and phylogeny of the MHC class I-related MR1 gene", Journal of immunolog, 161 (8), 4066-4077 (1998).

33. S. Huang, E. Martin, S. Kim, L. Yu, C. Soudais, D. H. Fremont, et al., "MR1 antigen presentation to mucosal-associated invariant $\mathrm{T}$ cells was highly conserved in evolution", Proceedings of the National Academy of Sciences of the United States of America, 106 (20), 8290-8295 (2009).

34. P. Boudinot, S. Mondot, L. Jouneau, L. Teyton, M.P. Lefranc and O. Lantz, "Restricting nonclassical MHC genes coevolve with TRAV genes used by innate-like $\mathrm{T}$ cells in mammals", Proceedings of the National Academy of Sciences of the United States of America, 113 (21), 2983-2992 (2016).

35. L. Le Bourhis, E. Martin, I. Péguillet, A. Guihot, N. Froux, M. Coré, et al., "Antimicrobial activity of mucosalassociated invariant T cells", Nature immunology, 11(8), 701- $\vee \cdot 8$ (2010).

36. N. A. Gherardin, M. N. Souter, H. F. Koay, K. M. Mangas, T. Seemann, T.P. Stinear, et al., "Human blood MAIT cell subsets defined using MR1 tetramers", Immunology and cell biology, 96 (5), 507-525 (2018).

37. M.e.a.H.M.c.a.x.-r. Dusseaux, "Human MAIT cells are xenobiotic-resistant, tissue-targeted, CD161hi IL-17-secreting $\mathrm{T}$ cells". Blood, 117 (4), 1250-1259 (2011).

38. O. Patel, L. Kjer-Nielsen, J. Le Nours, S. B. Eckle, R. Birkinshaw, T. Beddoe, et al., "Recognition of vitamin B metabolites by mucosal-associated invariant T cells", Nature communications, 4, 2142 (2013).

39. S. Mondot, P. Boudinot and O. Lantz, "MAIT, MR1, microbes and riboflavin: a paradigm for the co-evolution of invariant TCRs and restricting MHCI-like molecules?", Immunogenetics, 68 (8) 537-548 (2016).
40. E. Merlini, M. Cerrone, B. van Wilgenburg, L. Swadling, E.S. Cannizzo, A. d'Arminio Monforte, et al., "Association Between Impaired Va7.2+CD161++CD8+ (MAIT) and Va7.2+CD161-CD8+ T-Cell Populations and Gut Dysbiosis in Chronically HIVand/or HCV-Infected Patients", Frontiers in microbiology, 10, 1972 (2019).

41. N. A. Gherardin, A. N. Keller, R. E. Woolley, J. Le Nours, D. S. Ritchie, P. J. Neeson, et al., "Diversity of $\mathrm{T}$ Cells Restricted by the MHC Class I-Related Molecule MR1 Facilitates Differential Antigen Recognition", Immunity, 44 (1), 32-45 (2016).

42. J. Dias, E. Leeansyah and J. K. Sandberg, "Multiple layers of heterogeneity and subset diversity in human MAIT cell respo nses to distinct microorganisms and to innate cytokines", Proceedings of the National Academy of Sciences of the United States of America, 114 (27), 54345443 (2017).

43. D. I. Godfrey, H.F. Koay, J. McCluskey and N. A. Gherardin, "The biology and functional importance of MAIT cells", Nat Immunol, 20, 1110-1128 (2019).

44. A. J. Corbett, S.B. Eckle, R.W. Birkinshaw, L. Liu, O. Patel, J. Mahony, et al., "T-cell activation by transitory neoantigens derived from distinct microbial pathways", Nature, 509 (7500), 361-365 (2014).

45. R. Reantragoon, A. J. Corbett, I. G. Sakala, N. A. Gherardin, J. B. Furness, Z. Chen, et al., "Antigen-loaded MR1 tetramers define $\mathrm{T}$ cell receptor heterogeneity in mucosal-associated invariant $\mathrm{T}$ cells", The Journal of experimental medicine, 210 (11), 23052320 (2013).

46. J. Dias, C. Boulouis, J.B. Gorin, R. van den Biggelaar, K.G. Lal, A. Gibbs, et al., "The CD4(-) CD8(-) MAIT cell subpopulation is a functionally distinct subset developmentally related to the main CD8(+) MAIT cell pool", Proceedings of the National Academy of Sciences of the 
United States of America, 115 (49), 11513-11522 (2018).

47. H. F. Koay, N. A. Gherardin, A. Enders, L. Loh, L. K. Mackay, C. F. Almeida, et al., "A three-stage intrathymic development pathway for the mucosalassociated invariant $\mathrm{T}$ cell lineage", Nature immunology, 17 (11), 1300-1311 (2016).

48. E. Leeansyah, A. Ganesh, M.F. Quigley, A. Sönnerborg, J. Andersson, P .W. Hunt, et al., "Activation, exhaustion, and persistent decline of the antimicrobial MR1-restricted MAIT-cell population in chronic HIV-1 infection", Blood, 121 (7), 1124-1135 (2013).

49. H. Koppejan, D. Jansen, M. Hameetman, R. Thomas, R.E.M. Toes and F. A. van Gaalen, "Altered composition and phenotype of mucosal-associated invariant $\mathrm{T}$ cells in early untreated rheumatoid arthritis", Arthritis research \& therapy, 21(1), 1-7 (2019).

50. M. J. Sobkowiak, H. Davanian, R. Heymann, A. Gibbs, J. Emgård, J. Dias, et al., "Tissue-resident MAIT cell populations in human oral mucosa exhibit an activated profile and produce IL-17", European journal of immunology, 49(1), 133-143 (2019).

51. A. Rahimpour, H.F. Koay, A. Enders, R. Clanchy, S.B. Eckle, B. Meehan, et al., "Identification of phenotypically and functionally heterogeneous mouse mucosal-associated invariant $\mathrm{T}$ cells using MR1 tetramers", The Journal of experimental medicine, 212 (7), 1095108 (2015).

52. Y. Cui, K. Franciszkiewicz, Y.K. Mburu, S. Mondot, L. Le Bourhis, V. Premel, et al., "Mucosal-associated invariant $\mathrm{T}$ cellrich congenic mouse strain allows functional evaluation", The Journal of clinical investigation, 125(11), 4171-4185 (2015).

53. J. L. Croxford, S. Miyake, Y.Y. Huang, M. Shimamura, T. Yamamura, "Invariant $\mathrm{V}$ (alpha)19i $\mathrm{T}$ cells regulate autoimmune inflammation", Nature immunology, 7(9), 987-994 (2006).

54. I. Kawachi, J. Maldonado, C. Strader, S. Gilfillan, "MR1-restricted V alpha 19i mucosal-associated invariant $\mathrm{T}$ cells are innate $\mathrm{T}$ cells in the gut lamina propria that provide a rapid and diverse cytokine response", Journal of immunology, 176 (3), 1618-1627 (2006).

55. I. G. Sakala, L. Kjer-Nielsen, C. S. Eickhoff, X. Wang, A. Blazevic, L. Liu, et al., "Functional Heterogeneity and Antimycobacterial Effects of Mouse Mucosal-Associated Invariant $\mathrm{T}$ Cells Specific for Riboflavin Metabolites", Journal of immunology, 195 (2), 587-601 (2015).

56. N. Seach, L. Guerri, L. Le Bourhis, Y. Mburu, Y. Cui, S. Bessoles, et al., "Double-positive thymocytes select mucosal-associated invariant $\mathrm{T}$ cells", Journal of immunology, (Baltimore, Md. : 1950), 191(12), 6002-6009 (2013).

57. H. F. Koay, D. I. Godfrey and D. G. Pellicci, "Development of mucosalassociated invariant T cells", Immunology and cell biology, 96 (6), 598-606 (2018).

58. M. Salou, F. Legoux, J. Gilet, A. Darbois, A. du Halgouet, R. Alonso, et al., "A common transcriptomic program acquired in the thymus defines tissue residency of MAIT and NKT subsets", The Journal of experimental medicine, 216 (1), 133-151 (2019).

59. M. Gutierrez-Arcelus, N. Teslovich, A. R. Mola, R. B. Polidoro, A. Nathan, H. Kim, et al., "Lymphocyte innateness defined by transcriptional states reflects a balance between proliferation and effector functions", Nature communications, 10 (1), 687 (2019).

60. A. Toubal, I. Nel, S. Lotersztajn, A. Lehuen, "Mucosal-associated invariant $\mathrm{T}$ cells and disease", Nature reviews Immunology, 19 (10), 643-657 (2019).

61. F. Legoux, D. Bellet, C. Daviaud, Y. El Morr, A. Darbois, K. Niort, et al., "Microbial metabolites control the thymic development of mucosal-associated 
invariant T cells", Science, 366(6464), 494-499 (2019).

62. H. F. Koay, S. Su, D. Amann-Zalcenstein, S. R. Daley, I. Comerford, L. Miosge, et al., "A divergent transcriptional landscape underpins the development and functional branching of MAIT cells", Science immunology, 4 (41), (2019).

63. S. J. Winter, H. Kunze-Schumacher, E. Imelmann, Z. Grewers, T. Osthues and A. Krueger, "MicroRNA miR-181a/b-1 controls MAIT cell development", Immunology and cell biology, 97 (2), 190-202 (2019).

64. Z. Lukasik, D. Elewaut and K. Venken, "MAIT Cells Come to the Rescue in Cancer Immunotherapy?", Cancers, 12 (2), (2020).

65. E. Gracey, Z. Qaiyum, I. Almaghlouth, D. Lawson, S. Karki, N. Avvaru, et al., "IL-7 primes IL-17 in mucosal-associated invariant $\mathrm{T}$ (MAIT) cells, which contribute to the Th17-axis in ankylosing spondylitis", Annals of the rheumatic diseases, 75 (12), 2124-2132 (2016).

66. C. A. Braudeau, K. Néel, A. Herbreteau, G. Salabert, N. Rimbert, M. Martin, J. C.;Hémont, C. Hamidou and M. Josien, "R. Persistent deficiency of circulating mucosal-associated invariant $\mathrm{T}$ (MAIT) cells in ANCA-associated vasculitis", Journal of autoimmunity., 70, 73-79 (2016).

67. A. Mekinian, T. Mahevas, M. Mohty, V. Jachiet, S. Rivière, O. Fain, et al., "Mucosal-associated Invariant Cells are Deficient in Systemic Sclerosis", Scandinavian journal of immunology, 86 (4), 216-220 (2017).

68. J. J. Wang, C. Macardle, H. Weedon, D. Beroukas and T. Banovic, "Mucosalassociated invariant $\mathrm{T}$ cells are reduced and functionally immature in the peripheral blood of primary Sjögren's syndrome patients", European journal of immunology, 46 (10), 2444-2453 (2016).

69. E. von Seth, C.L. Zimmer, M. ReuterwallHansson, A. Barakat, U. Arnelo, A. Bergquist, et al., "Primary sclerosing cholangitis leads to dysfunction and loss of MAIT cells", European journal of immunology, 48 (12), 1997-2004 (2018).

70. N. E. Serriari, M. Eoche, L. Lamotte, J. Lion, M. Fumery, P. Marcelo, et al., "Innate mucosal-associated invariant $\mathrm{T}$ (MAIT) cells are activated in inflammatory bowel diseases", Clinical and experimental immunology, 176 (2), 266-274 (2014).

71. K. Haga, A. Chiba, T. Shibuya, T. Osada, D. Ishikawa, T. Kodani, et al., "MAIT cells are activated and accumulated in the inflamed mucosa of ulcerative colitis", Journal of gastroenterology and hepatology, 31(5), 965-972 (2016).

72. T. Nitta, J. R. Oksenberg, N. A. Rao and L. Steinman "Predominant expression of $\mathrm{T}$ cell receptor $\mathrm{V} \alpha 7$ in tumor-infiltrating lymphocytes of uveal melanoma", Science 249, 672-674 (1990).

73. M. Siegel, H. L. Holley, S. L. Lee, "Epidemiologic studies on systemic lupus erythematosus. Comparative data for New York City and Jefferson County, Alabama, 1956-1965", Arthritis and rheumatism, 13 (6), 802-811 (1970).

74. W. J. Fessel, "Systemic lupus erythematosus in the community. Incidence, prevalence, outcome, and first symptoms; the high prevalence in black women", Archives of internal medicine, 134 (6), 1027-1·35 (1974).

75. C. J. Michet, Jr., C. H. McKenna, L. R. Elveback, R. A. Kaslow and L. T. Kurland, "Epidemiology of systemic lupus erythematosus and other connective tissue diseases in Rochester, Minnesota, 1950 through 1979", Mayo Clinic proceedings, 60 (2), 105-113 (1985).

76. M. Petri, "Hopkins Lupus Cohort. 1999 update", Rheumatic diseases clinics of North America, 26 (2), 199-213 (2000).

77. L. B. Tucker, S. Menon, J. G. Schaller and D. A. Isenberg, "Adult- and childhoodonset systemic lupus erythematosus: a comparison of onset, clinical features, serology, and outcome", British journal of rheumatology, 34 (9), 866-872 . 
78. L. Massardo, M. E. Martínez, S. Jacobelli, L. Villarroel, H. Rosenberg and S. Rivero, "Survival of Chilean patients with systemic lupus erythematosus", Seminars in arthritis and rheumatism, 24 (1), 1-11 (1994).

79. M. Abu-Shakra, M.B. Urowitz, D.D. Gladman and J. Gough, "Mortality studies in systemic lupus erythematosus. Results from a single center. II. Predictor variables for mortality", The Journal of rheumatology, 22(7), 1265-1270 (1995).

80. G. K. Lam and M. J. C. Petri," Assessment of systemic lupus erythematosus", E. Rheumatology",23(5), 120-132 (2005).

81. P. Enghard, C. Rieder, K. Kopetschke, J. R. Klocke, R. Undeutsch, R. Biesen, et al., "Urinary CD4 $\mathrm{T}$ cells identify SLE patients with proliferative lupus nephritis and can be used to monitor treatment response", Annals of the rheumatic diseases, 73 (1), 277-283 (2014).

82. J. E. Craft, "Follicular helper T cells in immunity and systemic autoimmunity", Nature reviews Rheumatology, 8 (6), 337347 (2012).

83. L. J. Xu H, Cui X, et al., "Increased frequency of circulating follicular helper $\mathrm{T}$ cells in lupus patients is associated with autoantibody production in a CD40Ldependent manner", Cell Immunol, 295 (1), 46e51 (2015).

84. T. Koga and G. C. Tsokos . "T cells and IL-17 in lupus nephritis", Clinical Immunology, 185, 95-99 (2017)

85. A. Laurence, C. M. Tato, T. S. Davidson, Y. Kanno, Z. Chen, Z. Yao, et al., "Interleukin-2 signaling via STAT5 constrains T helper 17 cell generation", Immunity, 26 (3), 371-381 (2007).

86. D. Comte, M. P. Karampetsou, K. KisToth, N. Yoshida, S. J. Bradley, V. C. Kyttaris, et al., "Brief Report: CD4+ T Cells From Patients With Systemic Lupus Erythematosus Respond Poorly to Exogenous Interleukin-2", Arthritis \& rheumatology, 69 (4), 808-813 (2017).

87. A. Chiba, R. Tajima, C. Tomi, Y. Miyazaki, T. Yamamura and S. Miyake,
"Mucosal-associated invariant $\mathrm{T}$ cells promote inflammation and exacerbate disease in murine models of arthritis", Arthritis and rheumatism, 64 (1), 153161 (2012).

88. T. N. Chiba A, Yoshikiyo K, Murayama G, Kitagaichi M, Yamaji $\mathrm{K}$, et al., "Activation status of mucosal-associated invariant $\mathrm{T}$ cells reflects disease activity and pathology of systemic lupus erythematosus", Arthritis Research \& Therapy, 19,58,1257-1265 (2017).

89. S. I. van Wilgenburg B, Hutchinson EC, Leng T, Kurioka A, Kulicke C, et al., "MAIT cells are activated during human viral infections", Nature Communications, 7,11653. doi: 10.1038/ncomms11653 (2016).

90. B. M. Ussher JE, Attwod E, Shadwell J, Richardson $\mathrm{R}$, de Lara $\mathrm{C}$, et al., "CD161++ CD8+ $\mathrm{T}$ cells, including the MAIT cell subset, are specifically activated by IL-12+IL-18 in a TCRindependent manner", European journal of immunology., 44(1),195-203 (2014).

91. K. H. Rahimpour A, Enders A, Clanchy R, Eckle SB, Meehan B, et al., "Identification of phenotypically and functionally heterogeneous mouse mucosal-associated invariant T cells using MR1 tetramers", The Journal of Experimental Medicine, 212(7),1095-1108 (2015).

92. Y. Miyazaki, S. Miyake, A. Chiba, O. Lantz and T. Yamamura, "Mucosalassociated invariant $\mathrm{T}$ cells regulate Th1 response in multiple sclerosis", International immunology, 23 (9), 529535 (2011).

93. A. Chiba, G. Murayama and S. Miyake, "Mucosal-Associated Invariant T Cells in Autoimmune Diseases", Frontiers in immunology, 9, 1333 (2018).

94. W. H. Chen Z, D'Souza C, et al., "Mucosal-associated invariant T-cell activation and accumulation after in vivo infection depends on microbial riboflavin synthesis and co stimulatory signals", Mucosal Immunol, 10,58-68 (2017). 
95. A. Kurioka, J. E. Ussher, C. Cosgrove, C. Clough, J.R. Fergusson, K. Smith, et al., "MAIT cells are licensed through granzyme exchange to kill bacterially sensitized targets", Mucosal immunology, 8 (2), 429-240 (2015).

96. B. van Wilgenburg, I. Scherwitzl, E. C. Hutchinson, T. Leng, A. Kurioka, C. Kulicke, et al., "MAIT cells are activated during human viral infections", Nature communications, 7, 11653 (2016).

97. L. Loh, Z. Wang, S. Sant, M. Koutsakos, S. Jegaskanda, A. J. Corbett, et al., "Human mucosal-associated invariant $\mathrm{T}$ cells contribute to antiviral influenza immunity via IL-18-dependent activation", Proceedings of the National Academy of Sciences of the United States of America, 113 (36), 10133-10138 (2016).
98. J. Jo, A.T. Tan, J.E. Ussher, E. Sandalova, X.Z. Tang, A. Tan-Garcia, et al., "Tolllike receptor 8 agonist and bacteria trigger potent activation of innate immune cells in human liver", PLoS pathogens, 10 (6), e1004210 (2014).

99. A. N. Keller, S. B. Eckle, W. Xu, L. Liu, V.A. Hughes, J.Y. Mak, et al., "Drugs and drug-like molecules can modulate the function of mucosal-associated invariant $\mathrm{T}$ cells", Nature immunology, 18 (4), 402411 (2017).

100. G. Murayama, A. Chiba, H. Suzuki, A. Nomura, T. Mizuno, T. Kuga, et al., "A Critical Role for Mucosal-Associated Invariant $\mathrm{T}$ Cells as Regulators and Therapeutic Targets in Systemic Lupus Erythematosus", Frontiers in immunology, 10, 2681 (2019). 


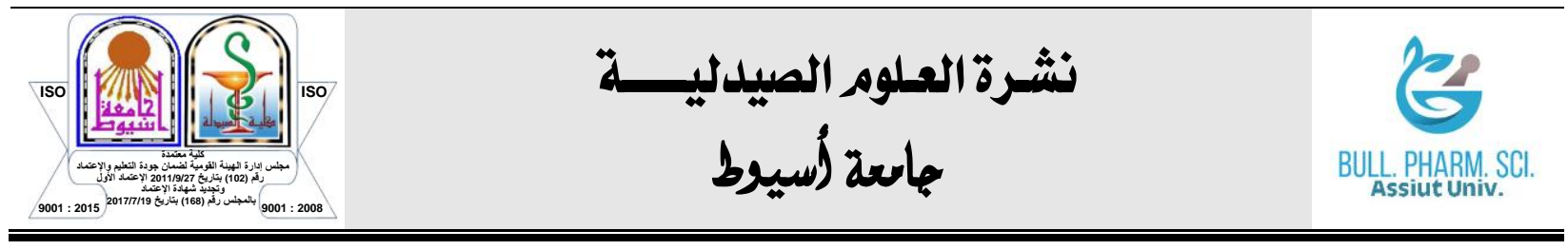

\section{دور الخلايا التائية المرتبطة بالغشاء المخاطي في الأبية الحمراء الجهازية}

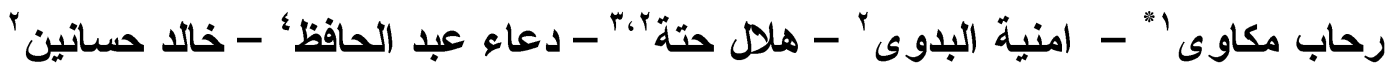

$$
\begin{aligned}
& \text { ' مصلحة الطب الشرعى ، وزارة العدل ، مصر }
\end{aligned}
$$

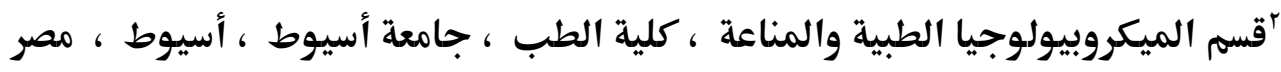

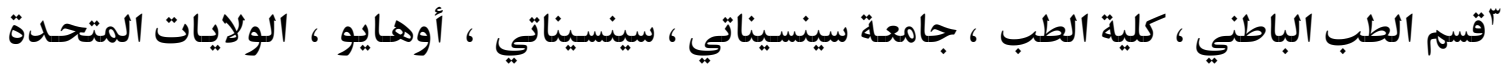

$$
\text { الأمريكية }
$$

عقم الطب الطبيعي وأمراض الروماتيزم والتأهيل ، كلية الطب ، جامعة أسيوط ، أسيوط ، مصر

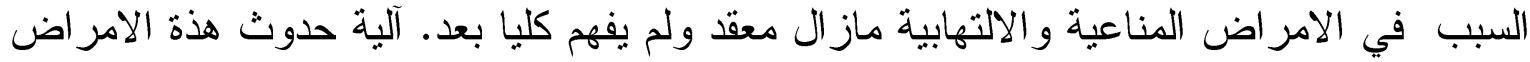

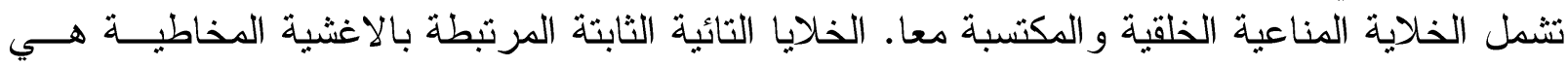

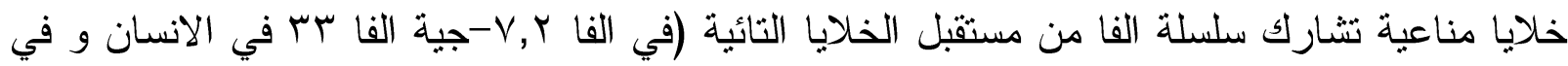

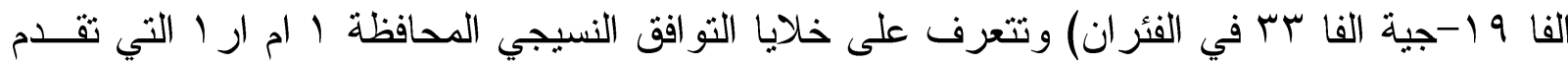

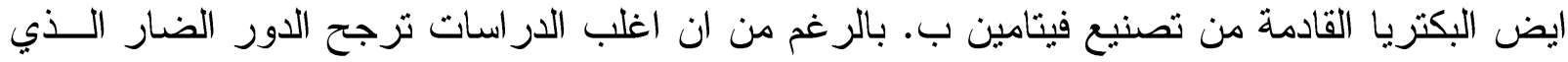

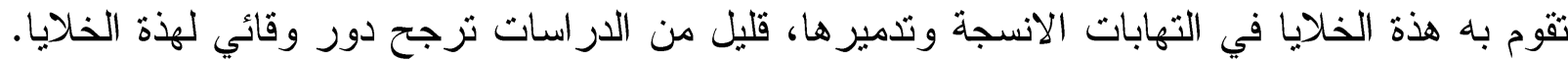

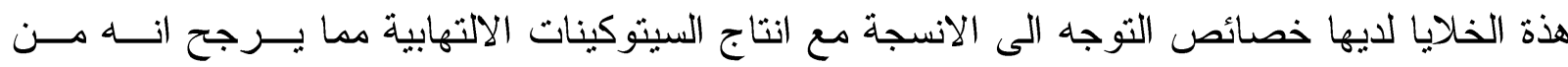

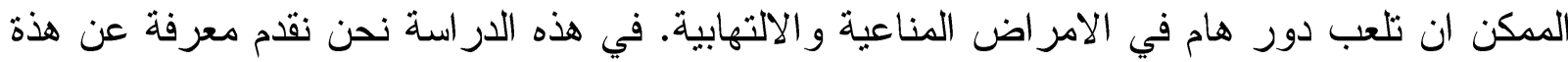

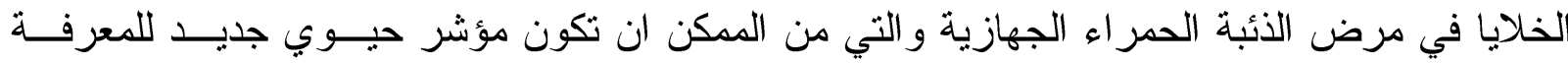
اكثربالمرض وي وضفتح تقنيات جديدة للعلاج. 